\title{
PROJETO de INTERVENÇÃo PARA CAPACITAÇÃo dAS EQUIPES dA ATENÇÃo PRIMÁRIA À SAÚdE PARA ACOLHIMENTTO DE PACIENTES EM SOFRIMENTO MENTAL NO MUNICIPIO DE NOVA ESPERANÇA
}

\author{
Intervention Project to train Primary Health Care \\ teams at embracement of mental illness patients in \\ the municipality of Nova Esperança
}

Anelise Ardengue Dias ${ }^{1}$, Clodoaldo Penha Antoniassi ${ }^{2}$

1. Especialista em Saúde Mental na Atenção Primária à Saúde, pela Escola de Saúde Pública do Paraná https://orcid.org/0000-0002-9220-5518

2. Mestrando do Programa de Pós-Graduação em Odontologia Integrada da Universidade Estadual de Maringá - UEM. https://orcid.org/0000-0001-9974-6088

CONTATO: Clodoaldo Penha Antoniassi | Rua Nivaldo Luiz Forastieri, 28 | Itambé | PR | CEP: 87175000 | Cel: (44) 988054007 | E-mail: cpaodonto@gmail.com

COMO CITAR Dias AA, Antoniassi CP. Projeto de Intervenção para capacitação das equipes da Atenção Primária à Saúde para acolhimento de pacientes em sofrimento mental no município de Nova Esperança. R. Saúde Públ. 2019 Jul.;2(Suppl 2): 113-124

(c) (i) COPYRIGHT Esta obra é disponibilizada nos termos da Licença Creative Commons - 4. 0

RESUMO A Atenção Primária à Saúde tem por objetivo realizar o atendimento inicial dos usuários no SUS, e a equipe encontra-se inserida no território, de forma que o vínculo entre a equipe e a população torna-se facilitado. Considerando que a porta de entrada para os diversos problemas de saúde da população é a atenção básica, e que 
entre os transtornos mentais, a maior parte da população que apresenta algum tipo de sofrimento mental não apresenta transtornos severos e persistentes, é importante ressaltar que esse sofrimento será acolhido na atenção básica. Sendo assim, é importante que toda a equipe das Unidades Básicas de Saúde esteja preparada para acolher tais demandas, com condições de fazer orientações e encaminhamentos se necessário. Assim, este projeto objetiva capacitar os profissionais da atenção básica para realizar o acolhimento de pacientes em situação de sofrimento mental.

PALAVRAS-CHAVE: Atenção Primária à Saúde. Saúde Mental. Capacitação de Recursos Humanos em Saúde.

ABSTRACT Primary health care is aimed at performing the initial care of users at the Unified Health System (SUS), and the team is inserted in the territory, so that the link between the team and the population becomes easier. Considering that the gateway to the population's various health problems is basic care, and that among mental disorders, the majority of the population that has some type of mental problem does not present severe and persistent disorders, it is important to note that this disorder will be embraced in basic care. Therefore, it is important that all the staff of the Basic Health Units be prepared to meet these demands, with the conditions to make guidelines and referrals if necessary. Thus, this project aims to enable primary care professionals to perform the care of mental disorders patients.

KEYWORDS: Primary Health Care. Mental Health. Health Human Resource Training.

\section{INTRODUÇÃO}

$\mathbf{A}$ atenção básica, ou atenção primária, é orientada pelos princípios da universalidade, acessibilidade, vínculo, continuidade do cuidado, integralidade da atenção, responsabilização, humanização, equidade e participação social. É a partir da atenção básica que as pessoas chegam até o SUS, inclusive aquelas em sofrimento mental. Neste nível de atenção, os profissionais podem conhecer melhor a realidade das pessoas e seus vínculos com a comunidade, pois as ações são desenvolvidas em um território geograficamente conhecido. Assim, o cuidado em Saúde Mental também pode ser considerado estratégico neste nível de atenção, devido à facilidade de acesso entre equipes e usuários?

O Movimento da Reforma Psiquiátrica ocorreu na década de 70, com a mobilização de familiares e profissionais de saúde, que buscavam mudar a realidade dos manicômios, promovendo a desinstitucionalização de pacientes, trazendo a necessidade de reordenar o modelo de atenção à saúde mental no Brasil. São criados então os serviços substitutivos ao modelo manicomial, como os Centros de Atenção Psicossocial (CAPS), os Serviços Residenciais Terapêuticos, entre outros. Além desses serviços, as Unidades Básicas de Saúde também desempenham função 
essencial dentro da rede de atenção à saúde mental, visto que estão inseridas no território, próximas à população, e as equipes possuem vínculos com os usuários. Através de intervenções próprias do processo de trabalho das equipes, pode-se considerar que os profissionais de saúde têm atitudes que possibilitam oferecer suporte emocional a pacientes em situação de sofrimento, como exercer boa comunicação, ser empático, escutar o usuário, acolher suas queixas como legítimas, entre outras?.

\section{JUSTIFICATIVA}

A Estratégia Saúde da Família (ESF) constitui-se como importante modelo de atenção, pois visa o atendimento à população em seu território, assistindo às necessidades de cada um. Também na atenção à saúde mental, a ESF tem se mostrado importante, possibilitando uma aproximação entre a família e o usuário, os profissionais da atenção básica e toda a comunidade?

Atualmente, cerca de 10 a 12\% da população necessitam de cuidados em saúde mental, porém não apresentam transtornos severos e persistentes, assim como 12\% da população acima de 12 anos apresentam transtornos decorrentes do uso de álcool e outras drogas. O cuidado a essa população é realizado pela atenção básica, de forma que a demanda em relação à saúde mental está cada vez mais presente neste nível de atenção ${ }^{3}$.

Baseando-se nos princípios da Atenção Primária à Saúde e da ESF, pode-se considerar que este nível de atenção seria resolutivo para um grande número de problemas em saúde mental, através da realização de ações de prevenção e promoção à saúde mental. Entretanto, percebe-se, nos profissionais de saúde, um distanciamento entre o que é preconizado pelas políticas públicas e o que acontece na prática. Os profissionais encontram-se "despreparados para entender o universo da Saúde Mental"4. o que gera posturas inadequadas, como ações normatizadoras e hospitalocêntricas; ações discriminatórias, moralistas e repressivas; infantilização, medicalização do sofrimento psíquico, entre outras.

Torna-se necessário, então, desconstruir estigmas relacionados à saúde mental, como as ideias de segregação e exclusão do paciente, e o pensamento de que o transtorno mental é uma situação imutável, que a pessoa com transtorno é incapaz de se emancipar e gerenciar sua própria vida. Considerando o atual modelo de atenção à saúde mental, essa visão do paciente dificulta o processo de transformação da assistência, pois as potencialidades do portador de transtorno mental não são valorizadas, e qualquer possibilidade de reabilitação não é levada em consideração².

Com o aumento da demanda de atenção à saúde mental nas Unidades Básicas de Saúde, percebe-se entre os profissionais uma dificuldade em realizar acolhimento aos usuários com queixas de saúde mental, o que resulta em diversos encaminhamentos destes pacientes para serviços especializados, como única alternativa para solucionar tal dificuldade 3 .

Sendo assim, é importante que exista formação técnica continuada, a partir da vivência nos serviços, o que possibilitaria a redução do sofrimento causado pelo despreparo para lidar com essas questões e, consequentemente, ocasionaria melhoria no atendimento aos usuários e seus familiares ${ }^{4}$

Dessa forma, tal projeto justifica-se como possibilidade de auxiliar as equipes da atenção primária do município de Nova Esperança a lidar com as questões de saúde mental, 
sentindo-se parte da rede e tendo condições de realizar acolhimento e orientações aos pacientes que apresentam tais necessidades, e encaminhamentos quando for necessário.

\section{DIAGNÓSTICO SITUACIONAL}

O município de Nova Esperança localizase na região noroeste do Estado do Paraná, em um território de aproximadamente $402 \mathrm{~km}^{2}$. No último censo, realizado em 2010, a população era de 26.615 habitantes, sendo 48,9\% homens e 51,1\% mulheres. A maior parte da população (24.323 pessoas) vive em áreas urbanas ${ }^{5}$.

A porta de entrada para a Rede de Saúde Mental ocorre nas Unidades Básicas de Saúde (UBS), onde o paciente, por meio de encaminhamento ou por demanda espontânea, é atendido por uma Psicóloga, para a realização da Estratificação de Risco em Saúde Mental, instrumento que possibilita identificar a gravidade de cada caso, para direcionar ao cuidado mais adequado. A partir da estratificação, é possivel distribuir a demanda de saúde mental nos diferentes níveis de assistência, sendo eles: Estratificação de Baixo Risco (acompanhamento na UBS, em atendimentos breves ou grupos); Estratificação de Médio Risco (encaminhamento para atendimento psicológico individual e consulta com psiquiatra); Estratificação de Alto Risco (encaminhamento para atendimento no CAPS, de acordo com a necessidade) ${ }^{6}$. Pacientes dependentes de álcool e drogas são acompanhados pelo CAPS, independentemente do resultado da estratificação de risco.

Dessa forma, o atendimento inicial de pacientes de saúde mental, assim como a manutenção daqueles que já receberam acompanhamento especializado, são realizados pelas UBS, que contam com um profissional da Psicologia em um dia da semana. Entretanto, percebe-se entre os profissionais das unidades uma certa insegurança para lidar com esses pacientes, o que acaba gerando diversos encaminhamentos para a psicologia, sendo que, em alguns casos, qualquer trabalhador da equipe poderia realizar acolhimento desses pacientes.

Entre janeiro e novembro de 2017, no município de Nova Esperança, foram realizadas 424 Estratificações de Risco pelas psicólogas das UBS, sendo 260 adultos e 164 crianças e adolescentes. Deste total, $64,86 \%$ delas foram classificadas como baixo risco; $32,55 \%$, como médio risco; e 2,59\% como alto risco.

Com base nos dados apresentados, foi possivel perceber que, entre as demandas de saúde mental que chegam através das UBS, a maior parte dos atendimentos permanecem na própria unidade, enquanto uma minoria é encaminhada para outros serviços. Além disso, é possivel perceber a dificuldade entre os profissionais da Atenção Básica para a realização de acolhimento dos pacientes com algum tipo de sofrimento mental, visto que a atenção primária constitui-se como sendo a porta de entrada. Sendo assim, com este projeto, objetiva-se realizar uma intervenção com as equipes das unidades, a fim de auxiliá-las a lidar com as demandas de saúde mental, com condições de acolher e orientar os pacientes que apresentam tais demandas.

\section{OBJETIVOS}

O objetivo deste estudo consiste em capacitar as equipes da Atenção Primária à Saúde para realizar o acolhimento dos pacientes com algum tipo de sofrimento mental. 


\section{OBJETIVOS ESPECIFICOS}

- Promover informações sobre saúde e transtornos mentais, aos profissionais das Unidades Básicas de Saúde;

- Informar para as equipes da Atenção Primária à Saúde sobre o funcionamento da Rede de Saúde Mental no município;

- Sensibilizar os profissionais das UBS sobre a importância de realizar o acolhimento dos pacientes com demandas de saúde mental;

- Capacitar os profissionais das UBS para o acolhimento e atendimento aos pacientes com demandas de saúde mental, e realização dos devidos encaminhamentos, se necessário.

\section{REVISÃO DE LITERATURA REFORMA PSIQUIÁTRICA BRASILEIRA}

O movimento de reforma psiquiátrica brasileira teve início na década de 70 , quando começaram a surgir as denúncias de fraude e corrupção, e do abandono, violência e maustratos a que os pacientes internados nos hospícios eram submetidos?. Neste cenário, surge o Movimento dos Trabalhadores de Saúde Mental (MTSM), com papel relevante nas denúncias e acusações ao governo militar, e com reivindicações como aumento salarial, redução do excessivo número de consultas por turno de trabalho, críticas à cronificação do manicômio e ao uso de eletrochoque, melhores condições de assistência à população e humanização dos serviços ${ }^{8}$.

A partir dessas denúncias, e inspirado na Psiquiatria Democrática Italiana, liderada por Franco Basaglia, que promovia a desinstitucionalização através de estruturas de saúde mental que pudessem substituir a internação em hospícios, surge no Brasil o movimento de reforma psiquiátrica, almejando a substituição do modelo asilar pelo modelo psicossocial, que preconiza a utilização de dispositivos de reintegração sociocultural. Tal modelo considera o usuário como principal participante de seu tratamento, juntamente com a família e a comunidade?

Em 1987, ocorre a I Conferência Nacional de Saúde Mental, que apresenta novas propostas de cuidado. Em 1989, na cidade de Santos (SP), se desenvolve uma experiência que se aproxima dos pressupostos de Basaglia: é implantada uma rede pública de atenção territorial, com estrutura complexa, com capacidade para atender às demandas psiquiátricas, funcionando de forma substitutiva ao hospital psiquiátrico. Naquele mesmo ano, o Deputado Paulo Delgado apresenta o Projeto de Lei no 3.657/89, no qual propõe a extinção progressiva dos manicômios e sua substituição por outros modelos assistenciais $^{10}$.

Apesar da lei ter sido aprovada apenas no ano de 2001, o envio do projeto possibilitou que as discussões sobre o tema no país inteiro fossem intensificadas, e estimulou a criação de leis estaduais, em sete estados e no Distrito Federal, que regulamentaram a assistência não asilar. Também a partir do projeto, o Ministério da Saúde editou 11 portarias que, em conjunto, possibilitaram o controle dos hospitais psiquiátricos em funcionamento e incentivo para criação de novos serviços de assistência em todo o país?

No ano de 2001, é promulgada a Lei $n^{0}$ 10.216, com base no projeto de lei $n^{\circ} 3.657 / 89$, que ficou conhecida como a Lei da Reforma Psiquiátrica, que redireciona o modelo de assistência em saúde mental e dispõe sobre os 
direitos dos portadores de transtorno mental. Também em 2001 é realizada a III Conferência Nacional de Saúde Mental, cujo relatório final apresenta propostas que visam à mudança do modelo assistencial e expansão da rede de serviços alternativos ${ }^{10}$.

Dessa forma, atualmente a reforma psiquiátrica está inserida nas políticas de saúde, em nível federal, estadual e municipal, e não se restringe à substituiç̧ão do hospital psiquiátrico, mas objetiva a desinstitucionalização; ou seja, o desafio é que ocorra um real deslocamento das práticas psiquiátricas para práticas de cuidado na comunidade"1.

\section{PROCESSO DE TRABALHO NA ATENÇÃO BÁSICA}

O termo Atenção Primária à Saúde (APS), ou Atenção Básica $(A B)$, refere-se à atenção ambulatorial não especializada, realizada em unidades de saúde de um determinado sistema, oferecendo atividades clínicas de baixa complexidade. A APS proporciona o primeiro contato da população com o sistema de saúde, oferecendo solução para grande parte dos problemas apresentados; além de acompanhamento daquelas situações que precisam de atendimento mais especializado ${ }^{12}$.

Em 2012, com o intuito de regulamentar as ações de atenção primária, é aprovada a Política Nacional de Atenção Básica, onde os termos "atenção primária à saúde" e "atenção básica" passam a ser considerados equivalentes. De acordo com esta Política, a atenção básica é caracterizada por um conjunto de ações de saúde. no âmbito individual e coletivo, que abrange: promoção e proteção da saúde, prevenção de agravos, diagnóstico, tratamento, reabilitação, redução de danos e manutenção da saúde. 0 objetivo da atenção básica é o desenvolvimento de atenção integral, de forma que possa impactar na situação de saúde e autonomia das pessoas, e nos determinantes e condicionantes de saúde das coletividades. A principal estratégia para consolidação e expansão da Atenção Básica está na Saúde da Família, que passa a ser o principal instrumento para organização da Atenção Básica ${ }^{13}$.

A Estratégia Saúde da Família deve atuar como porta de entrada prioritária do SUS, objetivando a resolução de cerca de $80 \%$ dos problemas de saúde mais prevalentes por meio da atenção primária, o que inclui também os problemas relacionados à saúde mental, de forma que o vínculo e diálogo entre atenção básica e saúde mental tornam-se muito importantes ${ }^{14}$.

Pode-se dizer, então, que a implementação da Estratégia Saúde da Família e dos novos serviços substitutivos em saúde mental, principalmente os Centros de Atenção Psicossocial (CAPS). indica o avanço da política de saúde mental do SUS, a partir da vinculação entre as equipes nos diversos níveis de atenção e do aumento da resolubilidade que os serviços substitutivos propiciam, com consequente resolução dos problemas de saúde da população ${ }^{15}$.

Devido à proximidade com a comunidade, comumente as equipes da atenção básica se deparam com problemas de saúde mental e situações de risco que comprometem os vínculos familiares, como alcoolismo e outras dependências, psicoses, prostituição infantil, exclusão social, violência, suicídios e suas tentativas, abuso de benzodiazepínicos, transtornos de humor e retardo mental. Por esse motivo, é preciso que a atenção básica esteja preparada para enfrentar essa realidade e as diversas formas de sofrimento psíquico ${ }^{14}$. 


\section{ACOLHIMENTO EM SAÚDE MENTAL}

Acolhimento e vínculo são dispositivos muito importantes na relação entre trabalhador e usuário, pois permitem a responsabilização compartilhada entre os sujeitos envolvidos, e consequente construção de autonomia. O acolhimento está relacionado com um atendimento de qualidade, pautado no respeito, diálogo e escuta qualificada, que possibilitam o estabelecimento de uma relação de confiança entre trabalhadores de saúde e usuários e seus familiares. O acolhimento proporciona a construção de uma nova prática em saúde, através de ações como receber e ouvir a população que procura o serviço, oferecendo respostas adequadas às demandas desde a recepção e atendimento individual ou coletivo, até o encaminhamento, retorno, remarcação e alta; ou seja, o acolhimento perpassa todo o processo de cuidado ${ }^{14}$.

O vínculo é considerado como algo que liga as pessoas, indica interdependência. Referese ao compromisso dos profissionais com os pacientes e vice-versa. O vínculo é a construção de laços afetivos entre trabalhadores e usuários, e significa receber bem os usuários e buscar resolutividade para seus problemas ${ }^{16}$.

Em relação ao atendimento à saúde mental na atenção básica, o acolhimento e o vínculo são dispositivos que possibilitam o cuidado integral, através do diálogo e construção de laços afetivos, de confiança e respeito entre os trabalhadores e os usuários. Dessa forma, tais dispositivos facilitam a resolubilidade dos problemas de saúde e propiciam a corresponsabilização entre a equipe e a população, possibilitando a melhoria na qualidade de vida da pessoa em sofrimento mental16.

\section{OBJ. ESPECIFICO 01: PROMOVER INFORMAÇÕES SOBRE SAÚDE E TRANSTORNOS MENTAIS AOS TRABALHADORES DA UBS}

\begin{tabular}{|c|c|c|c|c|c|}
\hline $\begin{array}{l}\text { Proposta de } \\
\text { Intervenção }\end{array}$ & \multicolumn{5}{|c|}{$\begin{array}{l}\text { Apresentação do projeto de intervenção; } \\
\text { Apresentação de informações detalhadas sobre o conceito de saúde e os } \\
\text { transtornos mentais mais comuns. }\end{array}$} \\
\hline $\begin{array}{l}\text { Duração total das } \\
\text { ações }\end{array}$ & 2 horas & Frequência & 1h/semana & Prazo execução & 15 dias \\
\hline Técnica & \multicolumn{5}{|c|}{ Roda de conversa } \\
\hline $\begin{array}{l}\text { Detalhamento das } \\
\text { ações }\end{array}$ & \multicolumn{5}{|c|}{$\begin{array}{l}\text { 1. Apresentação do projeto de intervenção } \\
\text { - Reunião para apresentação do projeto e seus objetivos, esclarecimento } \\
\text { de dúvidas, realização de adequações que se fizerem necessárias; } \\
\text { - Apresentação do cronograma previsto. }\end{array}$} \\
\hline
\end{tabular}




\begin{tabular}{|c|c|}
\hline \multirow{5}{*}{$\begin{array}{l}\text { Detalhamento das } \\
\text { ações }\end{array}$} & $\begin{array}{l}\text { 2. Apresentação de informações detalhadas sobre o conceito de saúde e } \\
\text { os transtornos mentais mais comuns }\end{array}$ \\
\hline & $\begin{array}{l}\text { - Proporcionar troca de informações entre os participantes sobre o que } \\
\text { cada um entende por saúde e transtornos mentais; }\end{array}$ \\
\hline & $\begin{array}{l}\text { - Oferecer informações detalhadas, em linguagem acessível, sobre o que } \\
\text { se considera como saúde, e quais são os transtornos mentais mais } \\
\text { comuns na sociedade; }\end{array}$ \\
\hline & $\begin{array}{l}\text { - Esclarecer sobre situações cotidianas, que podem desencadear } \\
\text { sofrimento, mas que não se caracterizam como transtorno mental; }\end{array}$ \\
\hline & - Esclarecer dúvidas; desmistificar preconceitos. \\
\hline
\end{tabular}

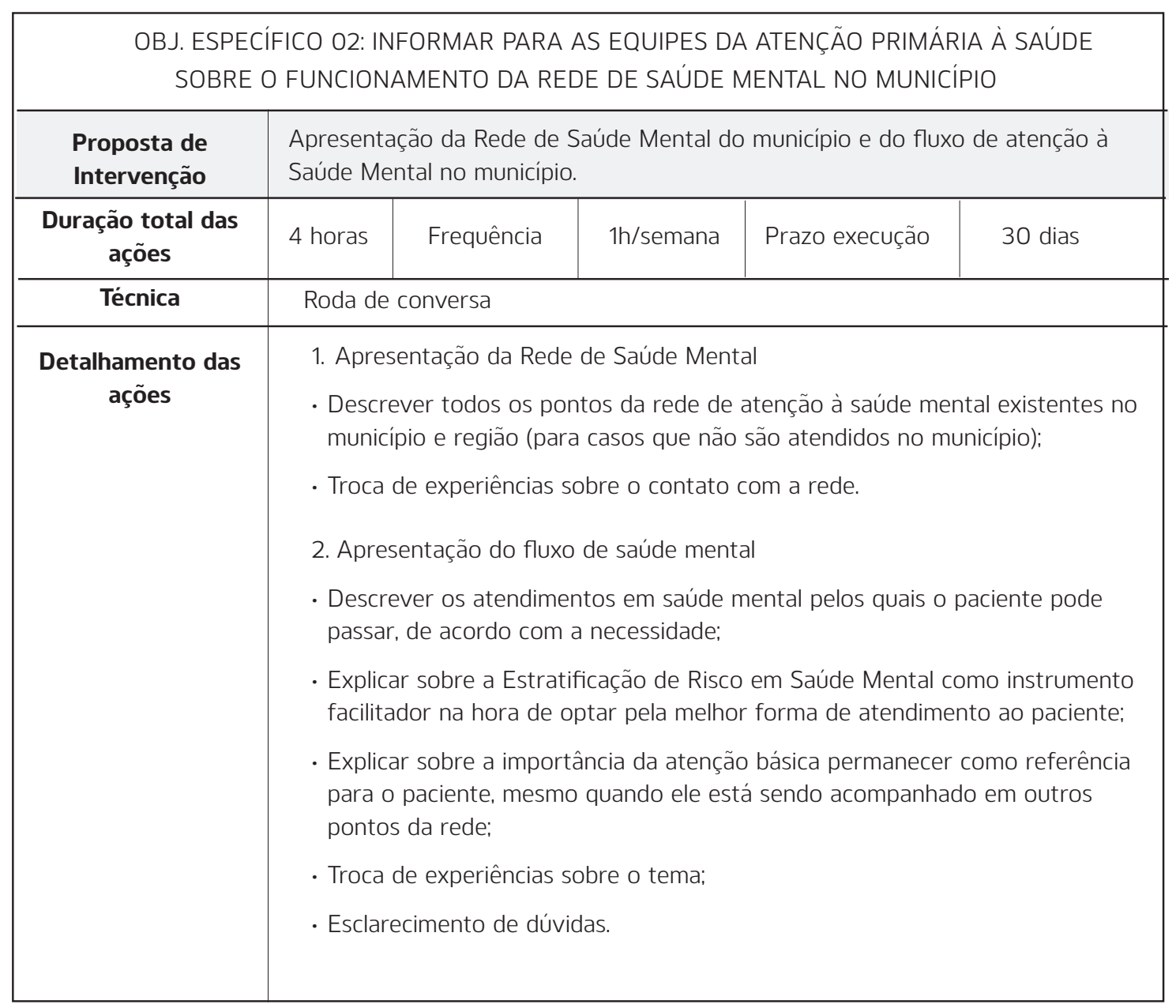


OBJ. ESPECÍFICO 03: SENSIBILIZAR OS PROFISSIONAIS DA UBS SOBRE A IMPORTÂNCIA DE REALIZAR O ACOLHIMENTO DOS PACIENTES COM DEMANDAS EM SAÚDE MENTAL

\begin{tabular}{|c|c|c|c|c|c|}
\hline $\begin{array}{l}\text { Proposta de } \\
\text { Intervenção }\end{array}$ & \multicolumn{5}{|c|}{$\begin{array}{l}\text { Apresentação da Rede de Saúde Mental do município e do fluxo de atenção à } \\
\text { Saúde Mental no município. }\end{array}$} \\
\hline $\begin{array}{l}\text { Duração total das } \\
\text { ações }\end{array}$ & 2 horas & Frequência & 1h/semana & Prazo execução & 15 dias \\
\hline Técnica & \multicolumn{5}{|c|}{ Roda de conversa } \\
\hline $\begin{array}{l}\text { Detalhamento das } \\
\text { ações }\end{array}$ & \multicolumn{5}{|c|}{$\begin{array}{l}\text { 1. Sensibilização para o acolhimento } \\
\text { - Conversa sobre as dificuldades no acolhimento às demandas de saúde mental; } \\
\text { - Esclarecimento de dúvidas a respeito do manejo de pacientes com alguma } \\
\text { queixa de saúde mental. }\end{array}$} \\
\hline
\end{tabular}

\begin{tabular}{|c|c|c|c|c|c|}
\hline \multicolumn{6}{|c|}{$\begin{array}{c}\text { OBJ. ESPECÍFICO 04: CAPACITAR OS PROFISSIONAIS DAS UBS PARA O ACOLHIMENTO } \\
\text { E ATENDIMENTO AOS PACIENTES COM DEMANDAS DE SAÚDE MENTAL, E REALIZAÇÃO DOS } \\
\text { DEVIDOS ENCAMINHAMENTOS, SE NECESSÁRIO. }\end{array}$} \\
\hline $\begin{array}{l}\text { Proposta de } \\
\text { Intervenção }\end{array}$ & \multicolumn{5}{|c|}{$\begin{array}{l}\text { Apresentação da Rede de Saúde Mental do município e do fluxo de atenção à } \\
\text { Saúde Mental no município. }\end{array}$} \\
\hline $\begin{array}{l}\text { Duração total das } \\
\text { ações }\end{array}$ & 8 horas & Frequência & 1h/semana & Prazo execução & 60 dias \\
\hline Técnica & \multicolumn{5}{|c|}{ Roda de conversa / Discussão } \\
\hline $\begin{array}{l}\text { Detalhamento das } \\
\text { ações }\end{array}$ & \multicolumn{5}{|c|}{$\begin{array}{l}\text { 1. Entendendo o acolhimento } \\
\text { - Discussão sobre o significado de acolhimento em saúde mental na atenção } \\
\text { básica; } \\
\text { - Conversa sobre a importância de realizar uma escuta atenta quando o usuário } \\
\text { fala sobre seus sentimentos; } \\
\text { - Orientações sobre as diferentes formas de acolher as demandas que surgem, } \\
\text { e a possibilidade de encaminhamento para atendimento especializado quando } \\
\text { houver necessidade; } \\
\text { - Esclarecimento de dúvidas. }\end{array}$} \\
\hline
\end{tabular}




\section{METODOLOGIA}

As ações a serem realizadas envolverão todos os trabalhadores das Unidades Básicas de Saúde, sempre levando em consideração as possibilidades de participação de cada um, de forma a não prejudicar o bom desempenho de suas funções. Para a realização das discussões, sugere-se a Roda de Conversa, metodologia que possibilita a troca de informações através de um espaço de diálogo entre os trabalhadores, onde eles podem escutar os outros e a si mesmos, resultando em reflexão sobre os assuntos abordados ${ }^{17}$.

Será elaborado um plano de ações para realizar a sensibilização dos diversos trabalhadores da atenção básica, para que eles possam compreender a importância do acolhimento em saúde mental; e capacitação para que todos se sintam seguros para realizar o devido acolhimento, assim como fazer orientações e os encaminhamentos necessários.

Serão necessários locais para o encontro com as equipes, e materiais para leitura/ informação sobre os temas propostos. Em relação aos recursos humanos, as capacitações serão realizadas pela equipe de saúde mental do município, e oferecidas às Equipes da Estratégia de Saúde da Família em cada UBS do município.

A seguir, apresentação das propostas de ação detalhadas de acordo com cada objetivo específico.

Em todas as ações, o responsável será o psicólogo/equipe de saúde mental, e o públicoalvo, todos os profissionais das UBS.

\section{ANÁLISE DA VIABILIDADE GERAL}

A realização deste projeto justifica-se com base em dados do município, onde é possível perceber a dificuldade dos trabalhadores da atenção básica em lidar com as demandas de saúde mental com as quais se deparam em seu cotidiano. Sendo assim, considera-se viável a execução deste projeto, por atender a uma necessidade apresentada pelos trabalhadores. A realização do projeto não requer investimento financeiro alto, visto que não há necessidade de contratação de recursos humanos adicionais, e o gasto com materiais será mínimo.

Pode-se considerar que algumas situações podem tornar inviável ou dificultar a realização do projeto, como a necessidade de realizar os encontros no horário de expediente, o que impossibilitaria a participação de todos os funcionários da unidade ao mesmo tempo. Nesta situação, pode-se considerar a possibilidade de fazer um rodízio dos participantes dos encontros, a fim de que todos possam participar sem que haja prejuízo no desempenho das funções essenciais de uma Unidade Básica de Saúde. Outra situação diz respeito à aceitação por parte dos trabalhadores, da realização do projeto, tendo em vista que alguns podem considerar como um acréscimo de função em suas atividades rotineiras.

\section{MONITORAMENTO E AVALIAÇÃO}

O monitoramento consiste em observação das ações planejadas e coleta de informações a respeito de sua execução, a fim de identificar possíveis falhas, possibilitando a sua correção ainda durante a execução do projeto, caso seja necessário. A realização do monitoramento permite que sejam observados aspectos relevantes, como os impactos das ações, alcance das metas, recursos empregados, aspectos 
facilitadores e limitadores, entre outros ${ }^{12}$. Para monitoramento das ações propostas, pretendese realizar, uma vez por mês, reuniões com a equipe, para verificar o andamento e alcance das metas.

A avaliação de um projeto tem por objetivo verificar se seus objetivos estão sendo alcançados, observando a efetividade do projeto no problema que ele se propõe a solucionar. Com base na avaliação do projeto, são tomadas decisões para aprimoramento das ações propostas, ou ainda para futuros projetos a serem implementados. A avaliação deve ser realizada em todas as fases do projeto, para confirmação da necessidade e importância da continuação do projeto ${ }^{12}$.

\section{RESULTADOS ESPERADOS}

Com a realização deste projeto, espera-se preparar as equipes das Unidades Básicas de Saúde para lidar com pacientes em sofrimento mental, sentindo-se capazes de acolher as demandas dessas pessoas, com uma escuta qualificada, e realizando encaminhamentos aos serviços especializados em saúde mental somente quando houver necessidade. Esperase que os profissionais das UBS possam reconhecer que algumas situações, que trazem sofrimento, são inerentes ao ser humano, e que também são passageiras, e, portanto, não necessitam de encaminhamento a algum serviço especializado. Dessa forma, pretende-se que ao longo do tempo, haja redução da lista de espera para acompanhamento psicológico na UBS. Além disso, espera-se que as equipes também possam reconhecer quando há a necessidade de uma intervenção pelo profissional ou equipe de saúde mental. Dessa forma, o conhecimento do funcionamento da Rede de Saúde Mental possibilita que os encaminhamentos sejam realizados adequadamente.

\section{CONSIDERAÇÕES FINAIS}

A atenção básica é considerada a porta de entrada para o Sistema Único de Saúde, tendo como ferramenta primordial a Estratégia Saúde da Família. É na atenção básica que os profissionais podem conhecer melhor a realidade da população, por estar inserida no território. Dessa forma, pela proximidade entre a equipe de saúde e a população, a atenção básica é responsável pela ordenação do cuidado em todos os níveis de atendimento, e em todos os aspectos inerentes ao ser humano, inclusive o sofrimento mental.

Considerando-se que atualmente, cerca de 10 a 12\% da população apresentam algum tipo de sofrimento mental, mas não apresentam transtornos severos e persistentes, e com base nos princípios da reforma psiquiátrica, de cuidado no território, pode-se afirmar que essa demanda é atendida pelas Unidades Básicas de Saúde, e a atenção primária pode ser resolutiva para um grande número dos problemas de saúde mental. Entretanto, percebe-se entre os profissionais da atenção básica, certa insegurança para lidar com essas demandas, o que acaba gerando diversos encaminhamentos aos serviços especializados.

Faz-se importante, portanto, que as equipes da atenção básica estejam preparadas para acolher as demandas de saúde mental que aparecem no território. Para isso, é preciso praticar o acolhimento, e ter um bom vínculo com a população. O acolhimento está 
relacionado ao atendimento de qualidade, com respeito, diálogo e construção de uma relação de confiança entre os envolvidos. O vínculo refere-se ao compromisso dos profissionais com os pacientes e vice-versa; é a construção de laços afetivos entre trabalhadores e usuários. Dessa forma, através do acolhimento e vínculo com a população, a equipe tem ferramentas para realizar o cuidado integral, inclusive às demandas de saúde mental, através de diálogo, confiança e respeito entre trabalhadores e usuários.

\section{REFERÊNCIAS}

.BRASIL. Ministério da Saúde. Secretaria de Atenção à Saúde. Departamento de Atenção Básica. Saúde mental / Ministério da Saúde, Secretaria de Atenção à Saúde, Departamento de Atenção Básica, Departamento de Ações Programáticas Estratégicas. Brasília: Ministério da Saúde, 2013. 176 p. (Cadernos de Atenção Básica, n. 34)

2.Pereira MAO. et al. Saúde Mental no Programa de Saúde da Família: conceitos dos agentes comunitários sobre o transtorno mental. Revista da Escola de Enfermagem da USP, 2007; vol. 41, n. 04, p. 567-572. Disponível em: < http://www.redalyc.org/ html/3610/361033292005/>. Acesso em: 11 set. 2017.

3.Sucigan DHI. Toledo VP, Garcia APRF. Acolhimento e saúde mental: desafio profissional na Estratégia Saúde da Família. Revista Rene, 2012; vol. 13, n. 01, p. 2-10. Disponivel em: <www.revistarene. ufc.br/revista/index.php/revista/article/download/10/7>. Acesso em: 06 nov. 2017.

4.Gama CAP. Campos RO. Saúde mental na atenção básica uma pesquisa bibliográfica exploratória em periódicos de saúde coletiva (1997-2007). Cadernos Brasileiros de Saúde Mental. 2009, out-dez, vol. 01, n. 02, p. 112-131. Disponivel em: < http:// incubadora.periodicos.ufsc.br/index.php/cbsm/article/view/1137>. Acesso em: 04 set. 2017.

5.IBGE - Instituto Brasileiro de Geografia e Estatística. IBGE Cidades. Disponivel em: <https://cidades.ibge.gov.br/>. Acesso em: 06 nov. 2017.

6.PARANÁ, Governo do Estado. A estratificação de risco em saúde mental. Coordenação Estadual de Saúde Mental. 2014. Disponivel em: < http://www.saude.pr.gov.br/arquivos/ File/APSUS_-_Ed._Permanente/Oficia_8___Saude_Mental/ Apresentacao_Estratificacao_de_Risco_em_Saude_Mental.pdf $>$. Acesso em: 21 nov. 2017.

7.Tenório F. A reforma psiquiátrica brasileira, da década de 1980 aos dias atuais: história e conceitos. Hist. Cienc. saúde-Manguinhos, Rio de Janeiro , v. 9, n. 1, p. 25-59, abr. 2002. Disponível em : <http://www.scielo.br/scielo.php?script=sci_arttext\&pid=S010459702002000100003\&lng=en\&nrm=iso>. Acesso em 13 abr.2018.

8.Luchmann LHH, Rodrigues J. O movimento antimanicomial no Brasil. Ciênc. saúde coletiva, Rio de Janeiro , v. 12, n. 2, p. 399-407, abr. 2007 . Disponivel em: <http://www.scielo.br/scielo. php?script=sci_arttext\&pid=S1413-81232007000200016\&lng=e n\&nrm=iso>. Acesso em 13 abr. 2018.
9.Duarte EOS et al. Caracterização das práticas de assistência na rede de atenção em saúde mental: revisão integrativa. Rev. Gaúcha Enferm., Porto Alegre, v. 33, n. 4, p. 191-199, Dez. 2012. Disponivel em: <http://www.scielo.br/scielo.php?script=sci_ arttext\&pid=S1983-14472012000400024\&lng=en\&nrm=iso >. Acesso em 17 ago. 2018.

10.Borges CF. Baptista TWF. O modelo assistencial em saúde mental no Brasil: a trajetória da construção política de 1990 a 2004. Cad. Saúde Pública, Rio de Janeiro, 24(2):456-468, fev, 2008. Disponivel em: <http://www.scielo.br/scielo.php?script=sci_ arttext\&pid=S0102-311X2008000200025>. Acesso em: 09 ago. 2018.

11.Aosani TR, Nunes KG. A saúde mental na atenção básica: a percepção dos profissionais de saúde. Rev. Psicol. Saúde, Campo Grande, v. 5, n. 2, p. 71-80, dez. 2013 . Disponivel em: <http:// pepsic.bvsalud.org/scielo.php?script=sci_arttext\&pid=S2177093X2013000200002\&lng=pt\&nrm=iso>. Acesso em: 04 mai. 2018.

12.LAVRAS, C. Atenção Primária à Saúde e a Organização de Redes Regionais de Atenção à Saúde no Brasil. Saúde Soc. São Paulo, v.20, n.4, p.867-874, 2011. Disponivel em: <http://www. scielo.br/pdf/sausoc/v20n4/05.pdf>. Acesso em: 05 jun. 18.

13.BRASIL. Ministério da Saúde. Política Nacional de Atenção Básica. Brasilia: Ministério da Saúde, 2012. (Série E. Legislação em Saúde)

14.Andrade W V; Botti NCL. A saúde mental na atenção básica articulação entre os princípios do sus e da reforma psiquiátrica. Cogitare Enfermagem, [S.I.], v. 13, n. 3, dez. 2008. ISSN 21769133. Disponível em: <https://revistas.ufpr.br/cogitare/article/ view/12991>. Acesso em: 13 abr. 2018

15.Morais APP. Tanaka OY. Apoio matricial em saúde mental: alcances e limites na atenção básica. Saude soc., São Paulo, v. 21, n. 1 , p. 161-170, Mar. 2012 . Disponivel em: <http://www.scielo.br/scielo. php?script=sci_arttext\&pid=S0104-12902012000100016\&lng=e n\&nrm=iso>. Acesso em 30 abr. 2018.

16.Jorge MSB et al. Promoção da Saúde Mental - Tecnologias do Cuidado: vínculo, acolhimento, co-responsabilização e autonomia. Ciênc. \& Saúde Coletiva, Rio de Janeiro , v. 16, n. 7. p. 30513060, Jul. 2011. Disponível em: <http://www.scielo.br/scielo. php?script=sci_arttext $\&$ pid=S1413-81232011000800005\&lng =e n\&nrm=iso>. Acesso em 20 abr. 2018.

17.Coelho DM. Intervenção em grupo: construindo rodas de conversa. In: Encontro Nacional da ABRAPSO, 14, 2007, Rio de Janeiro, Anais eletrônicos..., Rio de Janeiro: UFRJ: 2007. Disponivel em: <http://www.abrapso.org.br/siteprincipal/anexos/ AnaisXIVENA/conteudo/pdf/trab_completo_55.pdf>. Acesso em: 23 jul. 2018.

18.Barbosa RM. Monitoramento e Avaliação de Projetos Sociais. 2001. Disponivel em: <http://www.emater.tche.br/site/arquivos_ pdf/teses/mono_ricardo_barbosa.pdf >. Acesso em: 20 set. 2018. 